\title{
Collective quantum dot inversion and amplification of photon and phonon waves
}

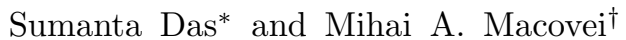 \\ Institute of Applied Physics, Academiei str. 5, MD-2028 Chişinău, Moldova \\ Max-Planck-Institut für Kernphysik, Saupfercheckweg 1, D-69117 Heidelberg, Germany
}

(Dated: June 25, 2018)

\begin{abstract}
The possibility of steady-state population inversion in a small sample of strongly driven two-level emitters like quantum dots (QDs) in micro-cavities, and its utilization towards amplification of light and acoustic waves is investigated theoretically. We find that inversion and absorption spectrum of photons, and phonons crucially depend on the interplay between the intrinsic vacuum and phonon environments. The absorption profiles of photons and phonons show marked novel features like gain instead of transparency and absorption reversed to gain, respectively. Furthermore, we report collectivity induced substantial enhancement of inversion and pronounced gain in the photon, and phonon absorption spectrum for a sub-wavelength-sized QD ensemble.

PACS numbers: 78.67.Hc, 43.35.Gk, 42.50.Ct, 42.50.Nn
\end{abstract}

\section{INTRODUCTION}

Even after fifty years of the advent of laser, achieving population inversion and lasing in novel systems remain a topic of continuing interest ${ }^{1}$. In particular, inversion in solid-state emitters like quantum dots (QDs) has attracted considerable interests recently ${ }^{2-5}$, due to the possibility of lasing in a driven two-level system 6,7 contrary to the common notion of multilevel manifolds 1 . Semiconductor QDs are novel nano-structures with unique engineerable features like large optical dipole moments and emission wavelengths. They can also be integrated efficient to micro-cavities and waveguides ${ }^{8}$. Their similarities to two-level atoms have been demonstrated in phenomena like Autler-Townes doublet ${ }^{9}$, Mollow triplet in resonance fluorescence ${ }^{10.11}$, spectral line narrowing ${ }^{12-14}$ and superradiance ${ }^{15}$. Furthermore, phonon-assisted excitation transfer in quantum dot molecules has been investigated in ${ }^{16}$. Additonally, due to potential application as solid state qubits numerous coherent optical studies of this system has been undertaken in the past decade ${ }^{17-22}$. During last few years, sensitivity of QDs to the nature of their phonon environment has been exploited in numerous investigations. Phonon-assisted damping of Rabi oscillations ${ }^{23-26}$, effect of phonons on polarization-entangled photons 27 , phonon induced transitions of excitons to cavity photons ${ }^{28}$, heat pumping with optically driven excitons in phonon environment ${ }^{29}$ and effect of electron-phonon coupling on resonance fluorescence spectrum ${ }^{30}-32$ have been reported. Moreover, phonon-mediated population inversion in a single QDcavity system driven by a continuous wave ${ }^{33}$ and a pulse laser excitation ${ }^{34}$ was proposed recently.

In light of these works, here, we propose a novel scheme to achieve and enhance population inversion in an ensemble of strongly driven two-level solid-state emitters like QDs via phonon-photon mediated collectivity (see Fig. 1). We find important interplay of the phononphoton reservoirs on the QD-dynamics and in creation of steady-state inversion in both bare- and dressedstates. As a key finding, we report phonon-induced unique features in the QD photon absorption spectrum like, gain instead of transparency at resonance and absorption switched to amplification for bare-state inversion. We also find amplification in the phonon absorption spectrum under condition of dressed-state inversion. Furthermore, for a single QD, the phonon emission exhibits anti-bunched phonon statistics, i.e. a quantum effect. Additionally, for a sub-wavelength-sized ensemble of QDs, we report phonon-assisted collective enhancement of inversion and improvement of gain in the photon or phonon absorption spectra.

The article is organized as follows. In Section II, we describe the analytical approach and the system of interest, and obtain the master equation characterising the photon-phonon mediated interaction among a collection of two-level pumped qubits. In Section III, we analyze

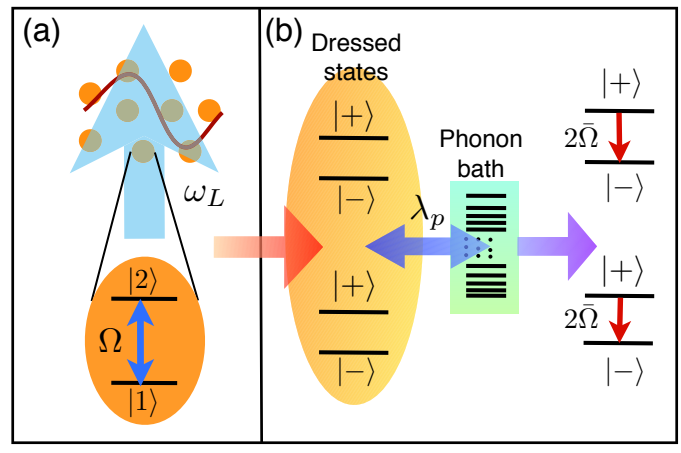

FIG. 1: (color online) Schematics of interaction of a collection of QDs with a coherent field and phonon reservoir. (a) QDs as two-level systems embedded in a substrate or in a microcavity of emission wavelength size, driven by a laser of Rabi frequency $\Omega$. (b) In strong field, the QDs are dressed by the laser giving rise to dressed-states $| \pm\rangle$ and characteristic Mollow triplet in emitted photon radiation. However, in presence of phonons, additional transitions at the Rabi frequency $2 \bar{\Omega}$ among the dressed-states are induced. 
the obtained results, respectively. The Summary is given in Section IV.

\section{SYSTEM OF INTEREST}

We consider an ensemble of QDs embedded in a substrate (such as InGaAs encased in GaAs substrate) or in a micro-cavity (like that formed by InAs/GaAs Bragg reflectors) illuminated by a cw laser of frequency $\omega_{L}$. As shown schematically in Fig. (11) the ground state $|1\rangle$ and the state containing a trapped electron-hole pair (exciton) $|2\rangle$ in each QD form a two-level system with transition frequency $\omega_{x}$. The linear dimension of the ensemble is smaller or of the order of the relevant emission wavelength. The laser is detuned from the exciton transition by $\Delta=\omega_{x}-\omega_{L}$ and drives it with a constant Rabi frequency of $\Omega$. Furthermore, the QDs interact with the solid state environment (phonon reservoir) and with field modes of the electromagnetic vacuum (photon reservoir) leading to different incoherent decay processes. The Hamiltonian describing the interaction of $N$ QDs with the laser and the vacuum and phonon reservoirs in a frame rotating with the laser frequency $\omega_{L}$ can be represented as:

$$
\begin{aligned}
\mathcal{H} & =\sum_{k} \hbar \delta_{k} a_{k}^{\dagger} a_{k}+\sum_{p} \hbar \omega_{p} b_{p}^{\dagger} b_{p}+\hbar \sum_{j=1}^{N} \Delta S_{j}^{z} \\
& +\hbar \sum_{j=1}^{N} \Omega\left(S_{j}^{+}+S_{j}^{-}\right)+\left\{i \sum _ { j = 1 } ^ { N } \left[\sum_{p} \lambda_{p} S_{j}^{+} S_{j}^{-} b_{p}^{\dagger}\right.\right. \\
& \left.\left.+\sum_{k}\left(\vec{g}_{k} \cdot \vec{\wp}_{j}\right) a_{k}^{\dagger} S_{j}^{-}\right]+ \text {H.c. }\right\} .
\end{aligned}
$$

In Eq. (10) the first three terms represent the free Hamiltonians of the electromagnetic vacuum, the phonon reservoir and the two-level QDs. The fourth term corresponds to the laser-QD interaction, while the two terms inside the curly brackets describe, respectively, the QDs interaction with the surrounding phonon and vacuum reservoirs. The photon/phonon operators satisfy the standard boson commutation relations: $\left[Q_{u}, Q_{u^{\prime}}^{\dagger}\right]=\delta_{u u^{\prime}}$ and $\left[Q_{u}, Q_{u^{\prime}}\right]=\left[Q_{u}^{\dagger}, Q_{u^{\prime}}^{\dagger}\right]=0,(Q=\{a, b\}, u=\{k, p\})$. The terms $\lambda_{p}, \vec{g}_{k}$ and $\vec{\wp}_{j}$ are, respectively, the QD-phonon coupling constant taken in a general form satisfying the weak-coupling limit, the mode function of the threedimensional multi-mode field, and the dipole moment of the $j$-th QD, while $\delta_{k}=\omega_{k}-\omega_{L}$. The raising and lowering operators for each QD are denoted by: $S_{j}^{+}=|2\rangle_{j j}\langle 1|$, $S_{j}^{-}=\left(S_{j}^{+}\right)^{\dagger}$ and $S_{j}^{z}=\left(|2\rangle_{j j}\langle 2|-| 1\rangle_{j j}\langle 1|\right) / 2$ while obeying the standard su(2) angular momentum commutation relations. In the following, we consider that the QDs system is driven by an intense laser. This leads to the dressing of both the bare-states in each QD creating the dressed-states:

$$
\begin{aligned}
|+\rangle_{j} & =\sin \theta|1\rangle_{j}+\cos \theta|2\rangle_{j}, \\
|-\rangle_{j} & =\cos \theta|1\rangle_{j}-\sin \theta|2\rangle_{j} .
\end{aligned}
$$

where $\tan 2 \theta=(2 \Omega / \Delta)$. Hence, it is imperative to continue the further analysis in the dressed-state basis.

The general form of the dressed master equation (DME) in the interaction picture is given by ${ }^{35}$ :

$$
\begin{aligned}
\partial \rho_{s b} / \partial t & =1 / i \hbar\left[\mathcal{H}_{I}(t), \rho_{s b}(0)\right] \\
& -1 / \hbar^{2} \int_{0}^{t} d t^{\prime}\left[\mathcal{H}_{I}(t),\left[\mathcal{H}_{I}\left(t^{\prime}\right), \rho_{s b}\left(t^{\prime}\right)\right]\right] .
\end{aligned}
$$

Here, $\rho_{s b}$ is the density operator of the combined QDs + reservoirs (both vacuum and phonons) in the interaction picture. The dressed-state interaction Hamiltonian $\mathcal{H}_{I}$ can be evaluated by using the dressed-state transformations (2) in Eq. (1) and an unitary transformation by the operator $U=e^{(i / \hbar) H_{0} t}$, where

$$
H_{0}=\sum_{k} \hbar \delta_{k} a_{k}^{\dagger} a_{k}+\sum_{p} \hbar \omega_{p} b_{p}^{\dagger} b_{p}+\hbar \bar{\Omega} \sum_{j=1}^{N} R_{j}^{z} .
$$

On substituting the interaction Hamiltonian and taking trace over the vacuum field and phonon modes, after some tedious algebra, we get the DME for the reduced density operator $\rho$ of the QDs in the Born-Markov and secular approximations, and weak coupling regime as:

$$
\begin{aligned}
\frac{\partial \rho}{\partial t} & +i \tilde{\Omega} \sum_{j}\left[R_{j}^{z}, \rho\right]=-\sum_{l, j}\left\{\chi_{0}\left[R_{l}^{z}, R_{j}^{z} \rho\right]\right. \\
& \left.+\chi_{+}\left[R_{l}^{+}, R_{j}^{-} \rho\right]+\chi_{-}\left[R_{l}^{-}, R_{j}^{+} \rho\right]\right\}+H . c .
\end{aligned}
$$

where $\tilde{\Omega}=\bar{\Omega}-\Delta_{p}$ with $\bar{\Omega}=\sqrt{(\Delta / 2)^{2}+\Omega^{2}}$ being the generalized Rabi frequency, while $\Delta_{p}$ is an additional shift due to QD-phonon coupling. The dressed basis operators are defined as: $R_{j}^{z}=|+\rangle_{j j}\langle+|-|-\rangle_{j j}\langle-|$ and $R_{j}^{+}=|+\rangle_{j j}\langle-|, R_{j}^{-}=\left(R_{j}^{+}\right)^{\dagger}$ obeying the commutation relations: $\left[R_{j}^{+}, R_{l}^{-}\right]=R_{j}^{z} \delta_{j l}$ and $\left[R_{j}^{z}, R_{l}^{ \pm}\right]= \pm 2 R_{j}^{ \pm} \delta_{j l}$.

In Eq. (4), $\chi_{0, \pm}$ are the renormalized decay rates given by:

$$
\begin{aligned}
\chi_{0} & =\left(\Gamma_{0} \sin ^{2} 2 \theta+\Gamma_{d} \cos ^{2} 2 \theta\right) / 4, \\
\chi_{+} & =\left[(\bar{n}+1) \Gamma_{p}+\Gamma_{d}\right] \sin ^{2} 2 \theta / 4+\Gamma_{+} \cos ^{4} \theta, \\
\chi_{-} & =\left(\bar{n} \Gamma_{p}+\Gamma_{d}\right) \sin ^{2} 2 \theta / 4+\Gamma_{-} \sin ^{4} \theta,
\end{aligned}
$$

respectively. Here, $\Gamma_{0}=(\mathcal{G} / \hbar)^{2} \kappa /\left[\kappa^{2}+\delta_{c}^{2}\right], \Gamma_{ \pm}=$ $(\mathcal{G} / \hbar)^{2} \kappa /\left[\kappa^{2}+\left(\delta_{c} \mp 2 \bar{\Omega}\right)^{2}\right], \quad \delta_{c}=\omega_{c}-\omega_{L}$ and $\Gamma_{p} \equiv$ $\Gamma_{p}(2 \bar{\Omega})=\pi \sum_{p}\left(\lambda_{p} / \hbar\right)^{2} \delta\left(\omega_{p}-2 \bar{\Omega}\right)$ with $\mathcal{G}=(\vec{g} \cdot \vec{\wp})$, while $\sin 2 \theta=\Omega / \bar{\Omega}, \cos ^{2} \theta=(\bar{\Omega}+\Delta / 2) / 2 \bar{\Omega}$, and $\sin ^{2} \theta=(\bar{\Omega}-$ $\Delta / 2) / 2 \bar{\Omega}$. Further, $\bar{n} \equiv \bar{n}(2 \bar{\Omega})=\left[\exp \left(2 \hbar \bar{\Omega} / k_{B} T\right)-1\right]^{-1}$ is the mean-phonon number at frequency $2 \bar{\Omega}$ and temperature $T$, with $k_{B}$ being the Bolzmann constant. The vacuum-induced dressed decay rates $\Gamma_{0, \pm}$ are derived under the assumption that the QDs interact with a broadband cavity mode of frequency $\omega_{c}$ and decay rate $\kappa . \Gamma_{p}$ is the phonon-induced dissipation rate of the QDs at the generalized Rabi frequency $2 \bar{\Omega}$ (see Fig. 1b) whereas $\Gamma_{d}$ describes the dephasing rate of $\mathrm{QDs}^{27}$. Notice that both 
reservoirs, i.e. photon and phonon, contribute to the collectivity among the QDs when fixed on a solid-state substrate. For this to occur, the inter-particle separations should be of the order of the smallest wavelength in the system (or less). Typically, the phonon wavelength in these samples is of the order of few/several nano-meters. Therefore, the Dicke limit considered here applies for a small QD system of an arbitrary shape or for specific geometries with moderate QDs numbers ${ }^{17}$. Indeed, when two or more qubits are close to each other on the emission wavelength scale then collective effects occur, i.e. the photon/phonon emission of one qubit influences its neighbor qubit and vice-versa. A small-sized QDs ensemble can be well within the Dicke-limit with respect to photon emission wavelength. For phonons the situation is different. The phonon emission wavelength in our system (the phonon linear dispersion $\omega_{p}=c_{s} p$ is a good approximation in the long wave vector limit; here, $c_{s}$ is the speed of sound while $p$ is the phonon wave vector) is of the order of few/several $\mathrm{nm}$ leading to inter-qubits phonon interactions only if the qubits's separation is of this order or less. This can be achieved, for instance, in grid-like patterns as it was the case for experimentally observation of photon- induced superradiance with quantum $\operatorname{dots}^{15}$ or in a quantum-dot array interacting via a common phonon bath $^{17}$. Here, however, the Dicke limit with respect to phonon wavelength may not be satisfied meaning that the collective decay rates due to phonon emission will depend on the inter-qubit separations, $r_{j l}=\left|\vec{r}_{j}-\vec{r}_{l}\right|$. However, for a few/several-qubit ensemble one can still consider that the collective decay rate is equal for all qubits. This happens because the inter-qubit separation in the collective decay rates enters as an argument to a sin/cos-like function ${ }^{17}$. Adjusting the inter-qubit separation with respect to the phonon wavelength one can avoid the inter-particle dependence of the phonon-induced decay rates for small-sized ensembles. Also, the exciton Bohr radius should be smaller than the size of a single QD, i.e. QDs are independent quantum objects in terms of quantum-mechanical tunnel coupling. Finally, in deriving Eq. (4), we have assumed that all dipole moments are equal, while for an inhomogeneous sample the frequency spread $\Delta \omega$ of the transition frequencies should be less than the collective decay rates, i.e. $\Delta \omega<N \chi_{0, \pm}$ (see, also, Ref.15).

In the following, we shall investigate the photonphonon mediated emission-absorption properties of an ensemble of two-level laser-pumped quantum dots described by Eq. (4).

\section{RESULTS AND DISCUSSION}

To understand the physics of QD dynamics, we next investigate the steady-state fluorescence and absorption spectrum of a single QD in the dressed basis and then generalize the obtained results to a multi-qubit ensemble.
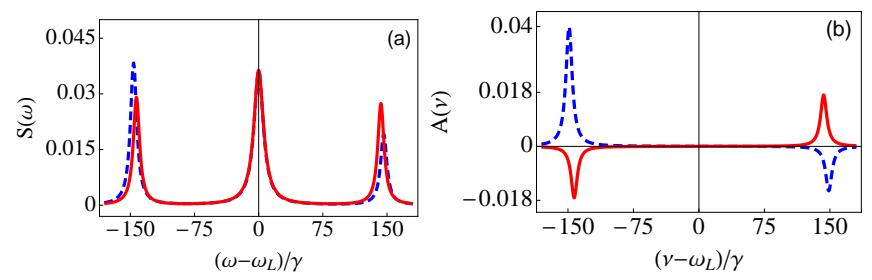

FIG. 2: (color online) The fluorescence and absorption spectrum of a $\mathrm{QD}$ at resonance (solid curve) and for negative detuning (dashed curve) of $\Delta=-40 \mu \mathrm{eV}$. The y-axis in both the plots are in dimensionless units and in (b) is multiplied by a factor of 10 . We have considered parameters similar to that achievable in experiments: $\Omega=80 \mu \mathrm{eV}, \Delta_{p}=20 \mu \mathrm{eV}$, $\Gamma_{0}=\Gamma_{ \pm}=\gamma=0.84 \mu \mathrm{eV}, \Gamma_{p}=0.34 \mu \mathrm{eV}, \Gamma_{d}=3.84 \mu \mathrm{eV}$ and $T=6 \mathrm{~K}$.

\section{A. Emission-absorption characteristics}

The total steady-state photon fluorescence spectrum ${ }^{35}$, in bad cavity limit and under secular approximation is evaluated as: $S_{t}(\omega)=S_{c}(\omega)+S(\omega)$, where $S_{c}(\omega)$ and $S(\omega)$ corresponds to the coherent and incoherent parts and for a single-qubit system are given by:

$$
S_{c}(\omega)=\frac{\pi}{4}\left\langle R^{z}\right\rangle_{s s}^{2} \sin ^{2} 2 \theta \delta\left(\omega-\omega_{L}\right)
$$

and, respectively,

$$
\begin{aligned}
& S(\omega)=\sin ^{4} \theta\left(\frac{\chi_{+}}{\chi_{+}+\chi_{-}}\right) \frac{\Gamma_{M L}}{\Gamma_{M L}^{2}+\left(\omega-\omega_{L}+2 \tilde{\Omega}\right)^{2}} \\
+ & \sin ^{2} 2 \theta \frac{\chi_{+} \chi_{-}}{\left(\chi_{+}+\chi_{-}\right)^{2}} \frac{\Gamma_{M C}}{\Gamma_{M C}^{2}+\left(\omega-\omega_{L}\right)^{2}} \\
+ & \cos ^{4} \theta\left(\frac{\chi_{-}}{\chi_{+}+\chi_{-}}\right) \frac{\Gamma_{M R}}{\Gamma_{M R}^{2}+\left(\omega-\omega_{L}-2 \tilde{\Omega}\right)^{2}}
\end{aligned}
$$

The corresponding photon absorption spectrum ${ }^{36}$ is:

$$
\begin{aligned}
A(\nu) & =i\left\langle R^{z}\right\rangle_{s s}\left\{\sin ^{4} \theta \frac{\Gamma_{M L}+i\left(\nu-\omega_{L}+2 \tilde{\Omega}\right)}{\Gamma_{M L}^{2}+\left(\nu-\omega_{L}+2 \tilde{\Omega}\right)^{2}}\right. \\
& \left.-\cos ^{4} \theta \frac{\Gamma_{M R}+i\left(\nu-\omega_{L}-2 \tilde{\Omega}\right)}{\Gamma_{M R}^{2}+\left(\nu-\omega_{L}-2 \tilde{\Omega}\right)^{2}}\right\},
\end{aligned}
$$

while for phonons is given by:

$$
A\left(\nu_{p}\right)=-\frac{i}{4}\left\langle R^{z}\right\rangle_{s s} \sin ^{2} 2 \theta\left\{\frac{\Gamma_{M L}+i\left(\nu_{p}-2 \tilde{\Omega}\right)}{\Gamma_{M L}^{2}+\left(\nu_{p}-2 \tilde{\Omega}\right)^{2}}\right\} .
$$

Here, $\left\langle R^{z}\right\rangle_{s s}$ is the single QD dressed basis steady-state population inversion given by: $\left(\chi_{-}-\chi_{+}\right) /\left(\chi_{-}+\chi_{+}\right)$. $\Gamma_{M L}=\Gamma_{R L}=4 \chi_{0}+\chi_{+}+\chi_{-}$and $\Gamma_{M C}=2\left(\chi_{+}+\chi_{-}\right)$ with $\left\{\nu, \nu_{p}\right\}$ being the weak photon and phonon probe frequencies, respectively.

In Fig. 2(a) \& 2(b), we plot Eq. (6) and imaginary part of Eq. (7). The solid (dashed) lines in the plot corresponds to resonant (non-resonant) coherent excitation 
of the QD. The photon fluorescence spectrum shown in Fig. 2(a) is similar to the Mollow spectrum well known for atoms, however, with certain key modifications due to the phonon bath. The position of the Mollow sidebands are shifted to $\pm 2 \tilde{\Omega}$ with phonon induced broadening of their widths $\Gamma_{M L}$ and that of the central line $\Gamma_{M C}$ as can be easily seen from their respective analytical forms. Additionally, in presence of phonons the sidebands are asymmetric with the ratio of the right to left peak heights as: $[(2 \bar{\Omega}+\Delta) /(2 \bar{\Omega}-\Delta)]^{2} \chi_{-} / \chi_{+}$. This asymmetry is pronounced for non-resonant driving of the QD as shown in Fig. 2(a) by the dashed curve. For resonant drive however, the asymmetry can be prominent in the conditions: low temperature reservoir and $\Gamma_{p} \gg\left(\Gamma_{d}, \gamma\right)$. The coherent part of the fluorescence spectrum $S_{c}(\omega)$, that signifies the elastic scattering process in presence of phonons, does not vanish even at resonance and has an amplitude $\propto \Gamma_{p}^{2} /\left[(2 \bar{n}+1) \Gamma_{p}+2\left(\Gamma_{d}+\gamma\right)\right]^{2}$ (see, also, Ref $\left.\frac{37}{37}\right)$. Note that, such phonon induced modifications of the Mollow triplet has been observed in a recent experiment ${ }^{32}$.

The imaginary part of the photon absorption spectrum shown in Fig. 2(b) is strikingly different from a typical Mollow absorption spectrum of a two-level atom. As a key finding, for resonant excitation of a $\mathrm{QD}$ we report phonon induced amplification and absorption in the spectrum at $\nu-\omega_{L}=\mp 2 \tilde{\Omega}$ respectively with peak heights $\propto\left\langle R^{z}\right\rangle_{s s} / 4$. Note that, these characteristics are absent in atomic system, where there is no phonon coupling and the probe field is transparent at resonance as $\left\langle R^{z}\right\rangle_{s s}=0$. Furthermore, for both resonant and non-resonant excitation, we find from Eq. (7) broadening of the amplification and absorption spectral lines due to phonons. The imaginary part of Eq. (8) exhibits another remarkable feature, gain in the phonon absorption spectrum for population inversion in the dressed-state. This, in principle, leads to amplification of a weak phonon wave in presence of an additional phonon cavity ${ }^{38,39}$ tuned to the dressed transition at the shifted generalized Rabi frequency of $2 \tilde{\Omega}$ (see Fig. 1b). Note that, the phonon amplification profile is enhanced only in presence of the vacuum reservoir as can be seen from the expressions for $\chi_{0, \pm}$.

For a collection of $N$ QDs in a sub-wavelength-sized ensemble, the emission-absorption spectrum is modified due to collective effects. The spectral lines are further broadened and enhanced, with the enhancement proportional to $N^{2}$ and $N$, respectively, for the Mollow peaks in photon fluorescence and gain profile in the photon or phonon absorption ${ }^{36}$. In the following, we discuss another key finding of the paper: collectivity assisted enhancement of steady-state population inversion.

\section{B. Enhanced inversion due to photon-phonon mediated collectivity}

In order to study the collective steady-state population inversion of the QDs in bare- and dressed-basis, we need to obtain the steady-state solution of Eq. (4). For this purpose, we first define the dressed-state collective operators as: $\mathcal{R}^{ \pm}=\sum_{j=1}^{N} R_{j}^{ \pm}$, and $\mathcal{R}^{z}=\sum_{j=1}^{N} R_{j}^{z}$, and then look for a solution of the form:

$$
\rho_{s s}=Z^{-1} \exp \left[-\eta \mathcal{R}^{z}\right]
$$

where the normalization $Z$ is determined by the requirement $\operatorname{Tr}\left\{\rho_{s}\right\}=1$. The unknown variable $\eta$ can be evaluated by inserting Eq. (9) in the DME and taking the steady-state $d \rho / d t=0$ condition. On doing so, we obtain: $\eta=\ln \left(\chi_{+} / \chi_{-}\right) / 2$. Note that, the coherent part of the dipole-dipole interaction potential commutes with the steady-state solution (9) obtained for a small-sized ensemble meaning that it does not modify the population distribution (unless it is larger than the generalized Rabi frequency). This can be seen by adding an extra term to the Hamiltonian (11), namely:

$$
H_{d d}=\Omega_{d d} \sum_{j \neq l=1}^{N} S_{j}^{+} S_{l}^{-},
$$

where the averaged over all possible orientation of dipoles coherent dipole-dipole interaction behaves as: $\Omega_{d d} \propto$ $1 /\left(2 r_{j l}\right)$, and it was taken identical for all involved qubits - an approximation valid for smaller ensembles. Applying the dressed-state transformation (2) to (10) in the secular approximation, one can observe that the Hamiltonian describing the dipole-dipole interaction includes the collective correlators: $R^{+} R^{-}, R^{-} R^{+}$and $R_{z}^{2}$, respectively, commuting with the solution (9). We next look for the inversion in the bare-states. In the secular approximation the collective bare-state inversion is given by:

$$
\left\langle\mathcal{S}^{z}\right\rangle_{s s}=(\Delta / 2 \bar{\Omega})\left\langle\mathcal{R}^{z}\right\rangle_{s s} / 2,
$$

where $\left\langle\mathcal{R}^{z}\right\rangle_{\text {ss }}$ is the collective steady-state dressed-state inversion found from Eq. (9) as ${ }^{7}$ :

$$
\left\langle\mathcal{R}^{z}\right\rangle_{s s}=N\left(\frac{1+e^{2 \eta(N+1)}}{1-e^{2 \eta(N+1)}}\right)+\frac{2\left(e^{2 \eta(N+1)}-e^{2 \eta}\right)}{\left(1-e^{2 \eta}\right)\left(1-e^{2 \eta(N+1)}\right)} .
$$

To gain physical insight into the behavior of the barestate population, we need to understand the inversion of dressed-state populations. For this purpose, we consider two limits in Eq. (12): (i) $e^{2 \eta}=\xi=\chi_{+} / \chi_{-} \ll 1$ and (ii) $\xi=\chi_{+} / \chi_{-} \gg 1$. From Eq. (12), we get in the limit (i): $\left\langle\mathcal{R}^{z}\right\rangle_{s s} / N \simeq 1-[2 \xi / N(1-\xi)]$, which clearly approaches maximum inversion $\left\langle\mathcal{R}^{z}\right\rangle_{s s} / N=1$, for $N \gg 1$. As $N$ represent the number of QDs in the ensemble, we can conclude that collectivity among the QDs enhances population inversion in the dressed-state. In the limit (ii) on the other hand, we get: $\left\langle\mathcal{R}^{z}\right\rangle_{s s} / N \simeq[2 / N(\xi-1)]-1$ which for $N \gg 1$ becomes, $\left\langle\mathcal{R}^{z}\right\rangle_{s s} / N=-1$, suggesting that all the population tends to reside in the lower dressed-state $|-\rangle$ and, thus, no inversion. Note that, in the limit $\chi_{+} / \chi_{-} \rightarrow 1,\left\langle\mathcal{R}^{z}\right\rangle_{s s} / N \rightarrow 0$ there is also no inversion as the dressed-states are equally populated. Additionally, we would like to emphasize that inversion is possible only in presence of the radiative decay $\Gamma_{ \pm}$among 

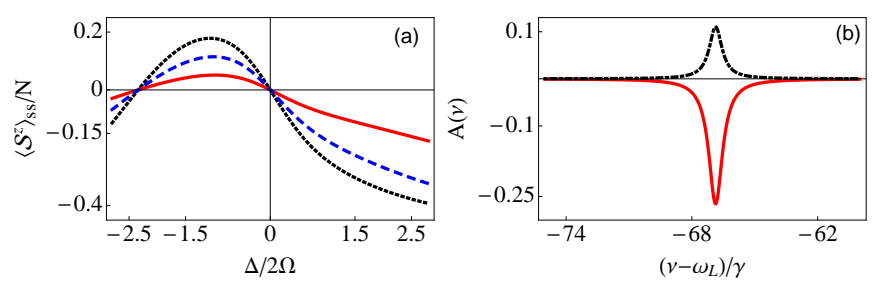

FIG. 3: (color online) (a) Steady-state population inversion in the bare-state for $N=10$ (dotted curve), $N=5$ (dashed line) and $N=1$ (solid curve) QDs. (b) Absorption spectrum of a single QD for $\Delta / 2 \Omega=-1$ (maximum inversion in Fig. 3a) in presence (solid curve) and absence (dot-dashed line) of phonons. In (b) the y-axis is in dimensionless units and multiplied by a factor of 100 while the $\mathrm{x}$-axis by 0.01 . Parameters for the plots are: $\Omega=2 \mathrm{meV}, \Delta_{p}=20 \mu \mathrm{eV}$, $\Gamma_{0}=\Gamma_{ \pm}=\gamma=0.84 \mu \mathrm{eV}, \Gamma_{p}=20 \gamma, \Gamma_{d}=40 \gamma$ and $T=6 \mathrm{~K}$.

the dressed-states with $\Gamma_{ \pm}>\Gamma_{p}$. This can be proved by analyzing the expressions for $\chi_{ \pm}$. On substituting $\Gamma_{ \pm}=0$ we have $\chi_{+} / \chi_{-}=\left[(\bar{n}+1) \Gamma_{p}+\Gamma_{d}\right] /\left[\bar{n} \Gamma_{p}+\Gamma_{d}\right] \geq 1$, for any bath temperature and QD parameters thereby implying no possible dressed-state inversion. Finally, setting $\left\{\Gamma_{ \pm}, \Gamma_{d}\right\}=0$ and $N=1$, one can recover the thermal population distribution among the dressedstates $\underline{40}$. Indeed, one can easily show that the steadystate upper and lower dressed-state populations (see, also, Fig. 11) are, respectively, $\left\langle R^{++}\right\rangle_{s s}=\bar{n} /(1+2 \bar{n})$ and $\left\langle R^{--}\right\rangle_{s s}=(1+\bar{n}) /(1+2 \bar{n})$. Their respective ratio is: $\left\langle R^{++}\right\rangle_{s s} /\left\langle R^{--}\right\rangle_{s s}=\bar{n} /(1+\bar{n}) \equiv e^{-\beta}$, where $\beta=\frac{2 \hbar \bar{\Omega}}{k_{B} T}$, and, thus, behaves according to thermal equilibrium relaxation. This will be the case also if spontaneous emission due to interaction with the vacuum modes of the surrounding electromagnetic field reservoir is smaller than the phonon-induced decay rates.

It is clear from the above discussion and Eqs (11) and (12), that for conditions of inversion in the dressed-state there will be no population inversion among the barestates $|1\rangle$ and $|2\rangle$. However, at low temperature (small $\bar{n})$, if $\Gamma_{p} \gg \Gamma_{ \pm}$we can satisfy the condition $\chi_{+} / \chi_{-} \gg 1$ and $\left\langle\mathcal{R}^{z}\right\rangle / N<0$ thereby leading to inversion among the bare-states for negative laser detuning. In Fig. 3(a), we show the steady-state population inversion among the bare-states for different number of QDs and typical experimental parameters $\underline{29}$. Inversion is seen to be achievable for certain range of negative laser detuning as expected from the above discussion. Additionally, we find collectivity induced enhancement of population inversion in the bare-state. This follows directly from the behavior of $\left\langle\mathcal{R}^{z}\right\rangle_{s s} / N$ for $\chi_{+} / \chi_{-} \gg 1$. We report almost $40 \%$ inversion for reasonable $(N=10)$ number of QDs (complete inversion in bare-state corresponds to a $\left.\left\langle S^{z}\right\rangle / N=0.5\right)$. Note that the mean-phonon number depends on the generalized Rabi frequency and, thus, varies as detuning varies. This has been explicitly considered here. In Fig. 3(b), we show the behavior of the imaginary part of photon absorption spectrum of Eq. (7) at maxi- mum bare-state inversion for a single QD. The spectrum show completely new feature in presence of phonons - amplification at bare-state inversion. However, for $\Gamma_{ \pm} \gg \Gamma_{p}$ or in absence of phonons, we get back the typical absorption peak instead of gain (dot-dashed line in Fig. $3 \mathrm{~b}$ ). This implies that we can get amplification of a probe laser from a QD ensemble.

Thus, concluding, for a small-sized ensemble of QDs, we find that collectivity enhances the inversion substantially both in bare and dressed-state. Hence, the phonon induced gain characteristics of photon-absorption spectrum that we report here for bare-state inversion, can be utilized towards amplification of a weak light field at optical frequencies in such solid-state systems. The photon induced gain characteristic of the phonon-absorption spectrum for dressed-state inversion, on the other hand, can amplify a phonon wave at $\mathrm{THz}$ frequencies tuned to the $2 \bar{\Omega}$ transition (as $\bar{\Omega} \sim$ ps is achievable in QDs). Furthermore, the phonon emission of a single pumped $\mathrm{QD}$ shows anti-bunched phonon statistics, namely:

$$
g^{(2)}(\tau)=1-\exp \left[-2\left(\chi_{+}+\chi_{-}\right) \tau\right]
$$

This can be understood as follows: once a phonon is emitted - the qubit will be in the lower dressed-state. Obviously, the qubit will generate then a photon on the dressed-state transition $|-\rangle \rightarrow|+\rangle$ or $|-\rangle \rightarrow|-\rangle$, respectively (see Fig. 11). Therefore, one can obtain in principle a flux of single-phonons. If the qubit is pumped with a strong pulse, then one can manage to obtain a singlephonon anti-bunched source. Here, one can look also at the photon-photon, phonon-phonon, phonon-photon or photon-phonon induced correlations. Thus, photonphonon correlations open interesting regimes of quantum statistics in these systems which can be harnessed towards quantum computation.

\section{SUMMARY}

Summarising, we have derived a collective dressedstate master equation for an ensemble of strongly driven QDs coupled to phonon and vacuum reservoirs. As a key finding, we reported gain characteristic in the photon and phonon absorption spectrum instead of transparency and absorption respectively due to crucial interplay of the vacuum and phonon reservoirs. Furthermore, we have shown that phonon mediated collectivity among the QDs enhances the population inversion in the bare-states. Anti-bunching occurs for phonon emission by a single pumped QD. Our findings show the possibility for amplification of photon and phonon waves as well as quantum particle correlations, and can open new directions towards creation of nanoscale optical and acoustic quantum or classical coherent sources. 


\section{ACKNOWLEDGEMENT}

We acknowledge insightful discussions with Prof. C. H. Keitel. S.D. is grateful for the hospitality of the
Institute of Applied Physics, Chişinău, Moldova, while M.M. acknowledges support via the research grant Nr. 13.820.05.07/GF.
* Electronic address: Sumanta.Das@mpi-hd.mpg.de

$\dagger$ Electronic address: macovei@phys.asm.md

1 P. W. Milloni and J. H. Eberly, Laser Physics (Wiley, New York, 2009).

2 T. M. Stace, A. C. Doherty, and S. D. Barrett, Phys. Rev. Lett. 95, 106801 (2005).

3 Y. Wu, I. M. Piper, M. Ediger, P. Brereton, E. R. Schmidgall, P. R. Eastham, M. Hugues, M. Hopkinson, and R. T. Phillips, Phys. Rev. Lett. 106, 067401 (2011).

4 E. R. Schmidgall, P. R. Eastham, and R. T. Phillips, Phys. Rev. B 81, 195306 (2010).

5 S. Hughes, and H. J. Carmichael, Phys. Rev. Lett. 107, 193601 (2011).

6 C. M. Savage, Phys. Rev. Lett. 60, 1828 (1988); T. Quang, and H. Freedhoff, Phys. Rev. A 47, 2285 (1993).

7 S. John, and T. Quang, Phys. Rev. Lett. 78, 1888 (1997).

${ }^{8}$ K. Hennessy, A. Badolato, M. Winger, D. Gerace, M. Atatüre, S. Gulde, S. Fält, E. Hu, A. Imamoglu, Nature 445, 896 (2007); M. Nomura, N. Kumagai, S. Iwamoto, Y. Ota, Y. Arakawa, Nature Physics 6, 279 (2010).

9 X. Xu, B. Sun, P. R. Berman, D. G. Steel, A. S. Bracker, D. Gammon, and L. J. Sham, Science 317, 929 (2007).

10 E. B. Flagg, A. Muller, J. W. Robertson, S. Founta, D. G. Deppe, M. Xiao, W. Ma, G. J. Salamo, and C. K. Shih, Nature Physics 5, 203 (2009).

11 A. N. Vamivakas, Y. Zhao, C.-Y. Lu, and M. Atatüre, Nature Physics 5, 198 (2009).

12 C. H. Keitel, Phys. Rev. Lett. 83, 1307 (1999).

13 O. Postavaru, Z. Harman, and C. H. Keitel, Phys. Rev. Lett. 106, 033001 (2011).

14 C. Matthiesen, A. N. Vamivakas, and M. Atatüre, Phys. Rev. Lett. 108, 093602 (2012).

15 M. Scheibner, Th. Schmidt, L. Worschech, A. Forchel, G. Bacher, Th. Passow, and D. Hommel, Nature Physics 3, 106 (2007).

16 E. Rozbicki, and P. Machnikowski, Phys. Rev. Lett. 100, 027401 (2008).

17 P. Zanardi, F. Rossi, Phys. Rev. Lett. 81, 4752 (1998).

18 W.W. Chow, H. C. Schneider, and M. C. Phillips, Phys. Rev. A 68, 053802 (2003).

19 G. X. Li, H. T. Tan, S. P. Wu, and Y. P. Yang, Phys. Rev. A 70, 034307 (2004).

${ }^{20}$ E. Paspalakis, M. Tsaousidou and A. F. Terzis, Phys. Rev.
B 73, 125344 (2006).

21 R. Hanson, D. D. Awschalom, Nature 453, 1043 (2008).

22 L.Yuan, and S. Das, Phys. Rev. A 83, 063819 (2011).

23 A. J. Ramsay, A. V. Gopal, E. M. Gauger, A. Nazir, B. W. Lovett, A. M. Fox, and M. S. Skolnick, Phys. Rev. Lett. 104, 017402 (2010).

24 J. Förstner, C. Weber, J. Danckwerts, and A. Knorr, Phys. Rev. Lett. 91, 127401 (2003).

25 A. Vagov, M. D. Croitoru, V. M. Axt, T. Kuhn, and F. M. Peeters, Phys. Rev. Lett. 98, 227403 (2007).

26 D. P. S. McCutcheon, N. S. Dattani, E. M. Gauger, B. W. Lovett, A. Nazir, Phys. Rev. B 84, 081305(R) (2011).

27 U. Hohenester, G. Pfanner, and M. Seliger, Phys. Rev. Lett. 99, 047402 (2007); S. Das, and G. S. Agarwal, J. Phys. B 41, 225502 (2008).

28 U. Hohenester, A. Laucht, M. Kaniber, N. Hauke, A. Neumann, A. Mohtashami, M. Seliger, M. Bichler, and J. J. Finley, Phys. Rev. B 80, 201311(R) (2009).

29 E. Gauger, J. Wabnig, Phys. Rev. B 82, 073301 (2010).

30 S. M. Ulrich, S. Ates, S. Reitzenstein, A. Löffler, A. Forchel, P. Michler Phys. Rev. Lett. 106, 247402 (2011).

31 C. Roy, S. Hughes, Phys. Rev. Lett. 106, 247403 (2011).

32 A. Ulhaq, S. Weiler, S. M. Ulrich, M. Jetter, P. Michler, C. Roy, and S. Hughes, Optics Express, 21, 4382 (2013).

33 S. Hughes, H. J. Carmichael, arxiv:1210:0488v1 (2013).

${ }^{34}$ D. E. Reiter, S. Lüker, K. Gawarecki, A. Grodecka-Grad, P. Machnikowski, V. M. Axt, and T. Kuhn, Acta Phys. Pol. A, 122, 1065 (2012).

35 G. S. Agarwal, Quantum Optics, (Cambridge University Press, 2012).

36 M. Macovei, J. Evers, and C. H. Keitel, Phys. Rev. Lett. 91, 233601 (2003); Phys. Rev. A 71, 033802 (2005).

37 D. McCutcheon, A. Nazir, Phys. Rev. Lett. 110, 217401 (2013).

38 M. Trigo, A. Bruchhausen, A. Fainstein, B. Jusserand, and V. Thierry-Mieg, Phys. Rev. Lett. 89, 227402 (2002).

39 J. Kabuss, A. Carmele, T. Brandes, and A. Knorr, Phys. Rev. Lett. 109, 054301 (2012).

40 M. Glässl, A. Vagov, S. Lüker, D. E. Reiter, M. D. Croitoru, P. Machnikowski, V. M. Axt, and T. Kuhn, Phys. Rev. B 84, 195311 (2011). 